\title{
Non-monotonic temperature evolution of dynamic correlations in glass-forming liquids
}

\author{
Walter Kob, ${ }^{1}$ Sándalo Roldán-Vargas,,${ }^{1,2}$ and Ludovic Berthier ${ }^{1}$ \\ ${ }^{1}$ Laboratoire Charles Coulomb, UMR CNRS 5221, \\ Université Montpellier 2, 34095 Montpellier, France \\ ${ }^{2}$ Departamento de Fisica Aplicada, Grupo de Fisica de Fluidos y Biocoloides, Universidad de Granada, 18071 Granada, Spain
}

(Dated: October 11, 2018)

\begin{abstract}
The viscosity of glass-forming liquids increases by many orders of magnitude if their temperature is lowered by a mere factor of $2-3^{1,2}$. Recent studies suggest that this widespread phenomenon is accompanied by spatially heterogeneous dynamics ${ }^{3,4}$, and a growing dynamic correlation length quantifying the extent of correlated particle motion ${ }^{5-7}$. Here we use a novel numerical method to detect and quantify spatial correlations which reveal a surprising non-monotonic temperature evolution of spatial dynamical correlations, accompanied by a second length scale that grows monotonically and has a very different nature. Our results directly unveil a dramatic qualitative change in atomic motions near the mode-coupling crossover temperature ${ }^{8}$ which involves no fitting or indirect theoretical interpretation. Our results impose severe new constraints on the theoretical description of the glass transition, and open several research perspectives, in particular for experiments, to confirm and quantify our observations in real materials.
\end{abstract}

More than forty years ago Adam and Gibbs $\underline{9}$ put forward the seminal idea that the relaxation dynamics of highly viscous liquids occurs through 'cooperatively relaxing regions'. Their theory suggested that particle motion occurs in a collective manner in localized domains ${ }^{9}$ whose typical size is related to the entropy of the systems and increases with decreasing temperature. This result implied that the relaxation mechanism is controlled by a unique length scale of thermodynamic origin with a direct signature in the dynamics. This approach regained momentum in the 1990's when novel experimental techniques and large scale computer simulations established the presence of dynamical heterogeneities, i.e. localized regions where dynamics is significantly different from the average ${ }^{3.4}$, although these observations can also be interpreted as a purely dynamical phenomenon 10 .

A qualitatively similar, but much more detailed, theoretical description is obtained within the framework of the random first order transition (RFOT) theory $\stackrel{11}{ }$. Within this approach, there exists an ideal glass transition that underlies glass formation, with an associated diverging correlation length scale of entropic origin since it is related to the existence of a large number of longlived metastable states. At very low temperatures, the glass-former is described as a 'mosaic' of correlated domains that rearrange in a thermally activated, collective manner, such that again static and dynamic correlations coincide and grow with the viscosity $\underset{12}{ }$. However, models with an RFOT (mainly mean-field like models) also display a 'spinodal' singularity at a higher temperature, $T_{c}$, at which metastability is lost and therefore the mosaic picture is no longer usefu111,13. Thus above $T_{c}$, a different approach must be used and it is found that the dynamics within mean-field models 14 has profound similarities with the one predicted from mode-coupling theory ${ }^{\underline{8}}$, where $T_{c}$ corresponds to a dynamic critical point associated with a diverging dynamical correlation length ${ }^{15}$. Thus, even at the theoretical level, the physics around $T_{c}$ remains 'mysterious'16: How can a dynamical correlation length diverge at two distinct temperatures? The mystery thickens in finite dimensions for which the mode-coupling singularity is cut off, and its existence can be inferred only from fitting relaxation data ${ }^{8}$, a procedure that is prone to criticisms, and therefore the physical relevance of the 'avoided' singularity at $T_{c}$ has remained a debated issue.

Progress is also slow because experiments on molecular systems do not have enough resolution to follow atomic motions over long times ${ }^{3}$, and numerical simulations often cannot access low enough temperatures to make definite statements on the size and nature of dynamic heterogeneities (in fact there are so far no numerical studies on the dynamical heterogeneities below $T_{c}$ ). At present, the most direct measurements $\mathrm{s}^{\frac{4}{}}$ seem to indicate that the dynamical correlation length increases from 1-2 particle diameters at moderately supercooled temperatures to 510 diameters close to the glass transition temperature, but the interpretation of the experimental data is often rather difficult $\underline{7}$. In this article we show that the use of a new methodology to characterize bulk dynamic correlations reveals details on their temperature evolution not observed in previous work.

In parallel to the quest for a dynamical length scale, evidence has also been found for an increasing of static correlations. However, this information is not captured by standard two-point correlation functions. Recent work has for instance suggested the growth of locally favored geometric structures in some model systems 17,18 , but these methods are not easily generalized to different glass-formers. One possibility to tackle this problem are the point-to-set correlations which are an elegant, general method to capture the multi-point static correlations which might characterize the non-trivial structure of viscous liquids 19 . The conceptual idea is to 'pin' the position of a number of particles (the 'set') in an equilibrated configuration of the fluid, and to measure how the position of the remaining particles is affected. It has recently been argued that in the geometry in which particles outside a spherical cavity are pinned, this point-to-set corre- 
lation should detect the typical domain size of the RFOT mosaic state ${ }^{20}$. Numerical simulations confirmed qualitatively the growth of point-to-set correlations in this particular geometry ${ }^{21}$. However, the connection to dynamic correlations and the precise temperature dependence of the various length scales were not studied, and these results did not resolve the 'mystery'16 of the $T_{c}$ crossover.

Inspired by previous work on confined fluids ${ }^{22}$ (see also Ref $\left.^{23}\right)$, we have generalized the idea of a point-to-set correlation to a novel geometry. We pin particles in a semi-infinite space and detect the resulting effect on the other half space. The principal advantage is that we can measure simultaneously the static and dynamic profiles induced by the frozen wall. Additionally we are able to perform simulations from the high temperature liquid down to and below $T_{c}$ with a realistic molecular dynamics, thus allowing us to resolve at once multi-point static and dynamic correlations in a very broad temperature regime encompassing the (hypothetical) mode-coupling crossover.

We study a binary mixture of quasi-hard spheres 24 , as described in the Methods. The fluid is equilibrated by means of standard molecular dynamics using periodic boundary conditions in all three directions. To simulate particles pinned within a semi-infinite space, $z<0$, it is enough to freeze at an arbitrary time $t=0$ the position of all particles within a slice of thickness $d_{\text {wall }}=1.4 \sigma$ which is perpendicular to the $z$-axis: They form our 'set'. Because we use periodic boundary conditions, we work with a very large system size in the $z$-direction, ensuring that bulk behavior is recovered at the center of the simulation box, i.e. the replicated walls do not interfere with each other.

To properly measure a point-to-set correlation it is crucial that the frozen walls have the same structure as the equilibrated liquid at temperature $T$, such that the average static properties of the confined liquid are unperturbed ${ }^{19,22}$ (see also Supplementary Material). We have measured how far (in $z$ ) the wall influences the static local density field and its dynamics, giving us independent access to static and dynamic correlation length scales. Note that within RFOT theory, the spatial extent of the static profile near a wall is not directly controlled by the mosaic length scale 13 , and a comparison with results obtained with a spherical cavity ${ }^{21}$ is not straightforward.

A convenient observable to characterize the influence of the wall is the overlap profile $q_{c}(t, z)$, defined as follows 21 . We discretize space into small cubic boxes of linear size $\delta \approx 0.55 \sigma$, and define $n_{i}(t)=1$ if box $i$ is occupied by at least one particle at time $t$, and $n_{i}(t)=0$ if not. The overlap profile in the $z$ direction with respect to the template configuration at time $t=0$ is

$$
q_{c}(t, z)=\left[\frac{\sum_{i(z)}\left\langle n_{i}(t) n_{i}(0)\right\rangle}{\sum_{i(z)}\left\langle n_{i}(0)\right\rangle}\right]_{\text {wall }},
$$

where the sums run over all boxes at distance $z$ from the wall, $\langle\cdots\rangle$ is the usual thermal average, and $[\cdots]_{\text {wall }}$ is
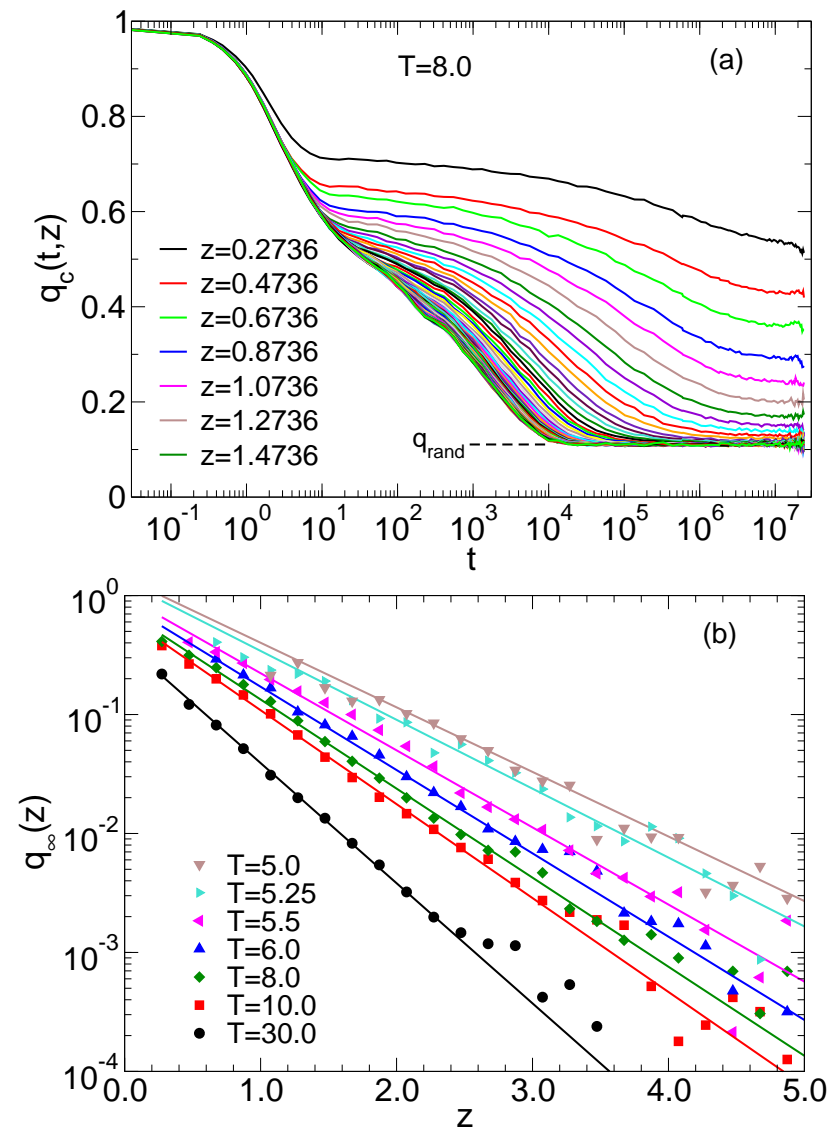

FIG. 1: (a) Time dependence of the overlap $q_{c}(t, z)$, Eq. (1), for $T=8.0$ and various values of $z$, increasing from $z=0.2736$ (near the wall, rightmost curve) by increments of 0.2 . The leftmost curve presents the bulk behavior. (b) $z$-dependence of the nontrivial part of the static overlap $q_{\infty}(z)$ for different temperatures (symbols). The solid lines are fits with the exponential given in Eq. (3).

an additional average over independent wall realizations. Thus $q_{c}(t, z)$ quantifies the similarity of particle configurations separated by a time $t$ at distance $z$, and, by construction, $q_{c}(t=0, z)=1$, for all $z$. We have also studied $q_{s}(t, z)$, the single particle version of Eq. (1), obtained by requesting that the box $i$ is occupied at times 0 and $t$ by the same particle. We find no relevant difference between these two correlation functions as far as time dependence is concerned.

In Fig. 11a we show the time dependence of $q_{c}(t, z)$ at $T=8.0$ for different values of $z$. For large $z$ (leftmost curves) the correlators become independent of $z$ and present the bulk behavior. The presence of a shoulder at intermediate times reflects the usual cage motion of particles observed in glassy systems ${ }^{2}$. In the long-time limit, $t \rightarrow \infty$, the correlator decays to $q_{\text {rand }}=0.110595$, the probability that a box is occupied, a quantity we measured with high precision from bulk simulations. With decreasing $z$, the height of the plateau at intermediate times increases and the timescale to reach it decreases. The final decay is much slower near the wall than in the 
bulk, showing that the $\alpha$-relaxation is strongly affected by the frozen wall. Furthermore the long-time limit of the overlap increases from its trivial value, $q_{c}(t \rightarrow \infty, z)>$ $q_{\text {rand }}$, showing that sufficiently close to the wall, the density cannot freely fluctuate. (See Supplementary Material for an illustration.) Thus the set of frozen particles with $z<0$ influences the position of the liquid particles at $z>0$ over a non-trivial static length scale. Finally, Fig. 19 shows that there exists a range of $z$ values for which the long-time limit of the overlap is the trivial bulk value, while the relaxation timescale is slower than the bulk. This directly shows, with no further analysis, that dynamic correlations have a larger range than static ones, as we confirm below.

To quantify these observations, we fit the final decay of $q_{c}(t, z)$ to the stretched exponential form

$$
q_{c}(t, z)-q_{\mathrm{rand}}=A \exp \left[-(t / \tau)^{\beta}\right]+q_{\infty},
$$

where $A, \tau, \beta$, and $q_{\infty}$ are fitted for each $z$. The profile of the static overlap, $q_{\infty}(z)$ measures how far from the wall density fluctuations are correlated, while $\tau(z)$ measures how far dynamics is affected. We find that Eq. (2) also describes well the single particle overlap, $q_{s}(t, z)$, with the obvious difference that $q_{s}(t \rightarrow \infty, z)=0$, because eventually all particles leave the box that they occupy at $t=0$. Thus, we obtain a second, 'self' dynamic profile from the study of $q_{s}(t, z)$.

In Fig. 1 $b$, we display the temperature evolution of the static overlap profiles $q_{\infty}(z)$. The semi-log plot suggests to describe these data using an exponential decay 25

$$
q_{\infty}(z)=B \exp \left(-z / \xi^{\text {stat }}\right),
$$

which allows us to define a static point-to-set correlation length scale $\xi^{\text {stat }}(T)$. From these data it is clear that $\xi^{\text {stat }}$ grows when temperature decreases, a result in good qualitative agreement with previous work ${ }^{21}$ using a very different geometry. Notice that $B(T)$ also changes rapidly with $T$, which suggests to define $\xi^{\text {stat-int }} \equiv B(T) \xi^{\text {stat }}$ as a convenient estimate of the integrated profile, $\xi^{\text {stat-int }} \approx$ $\int_{0}^{\infty} q_{\infty}(z) d z$.

We now analyze the dynamic profiles. To take into account the fact that the amplitude and stretching of the time dependent correlations evolve with $z$, see Fig. 1a, we have calculated the area under the correlators $q_{c}(t, z)$ and $q_{s}(t, z)$, taking into account only the secondary, slowly relaxing part. We denote the resulting times by $\tau_{c}(z)$ and $\tau_{s}(z)$, respectively. Previous studies ${ }^{22}$ have suggested that for large $z$ the $z$-dependence of $\tau_{s}(z)$ can be described well by an exponential functional form,

$$
\log \left(\tau_{s}\right)=\log \left(\tau_{s}^{\text {bulk }}\right)+B_{s} \exp \left(-z / \xi_{s}^{\text {dyn }}\right),
$$

where $B_{s}(z)$ and, more importantly, the dynamic length scale, $\xi_{s}^{\text {dyn }}(T)$, are adjusted for each $z$. The bulk relaxation time $\tau_{s}^{\text {bulk }}(T)$ is measured independently with a very good precision, see Fig. 2a. Using a power law fit inspired by mode-coupling theory $\underline{\underline{8}}, \tau^{\text {bulk }}(T) \sim\left(T-T_{c}\right)^{-\gamma}$, we obtain $T_{c} \approx 5.2$, but deviations from the algebraic fit
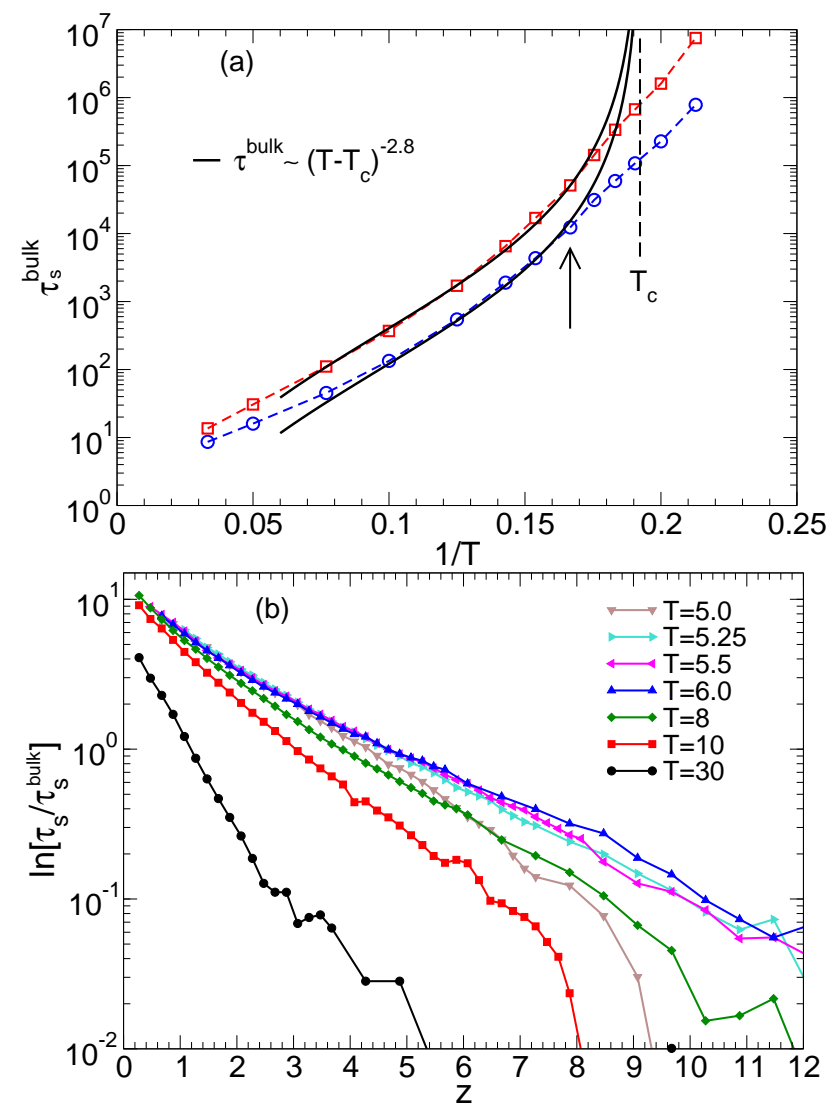

FIG. 2: (a) Temperature evolution of bulk relaxation time for large (top) and small (bottom) particles. Solid lines represent a power-law fit with $T_{c}=5.2$ (dashed line), the arrow indicates $T=6.0$ where deviations from the fit appear. (b) $z$-dependence of the relaxation time $\tau_{s}(z, T)$ at various temperatures. Note the non-monotonic $T$-dependence at intermediate and large $z$.

appear above $T_{c}$ near $T=6.0$, see Fig. 2a (see Supplementary Material for a discussion of this fit.)

In Fig. $2 \mathrm{~b}$ we show that the data at large $z$ can indeed be fitted well by the Ansatz given by Eq. (4). We have found a similar behavior for $\tau_{c}(z)$, providing us with a second dynamic correlation length scale, $\xi_{c}^{\text {dyn }}$. For high temperatures Eq. (44) gives a good description of the data over the entire range of distances $z$. For intermediate and low temperatures we see the development of a curvature in a semi-log plot, indicating at small $z$ deviations from the simple exponential dependence. This might suggest, although very indirectly, the appearance of more than one relaxation process for the relaxation dynamics.

A remarkable behavior occurs at intermediate and large distances, which has, to our knowledge, remained undetected. The dynamic profiles exhibit a striking nonmonotonic evolution with temperature. A close inspection of Fig. 2 $2 \mathrm{~b}$ shows that the dynamic profiles extend to increasingly larger distances when temperature decreases from $T=30.0$ down to $T=6.0$, but become shorterranged when $T$ is decreased further, down to $T=5.5$, 


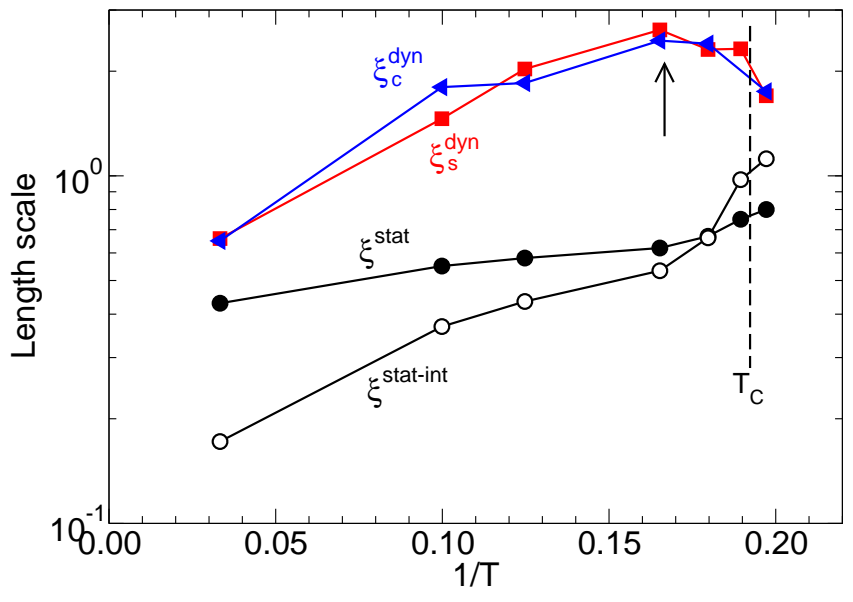

FIG. 3: Temperature dependence of static and dynamic length scales identified in this work. The arrow at $T=6.0$ and the dashed line at $T_{c}=5.2$ are as in Fig. 2. Dynamic length scales display a non-monotonic behavior with a maximum at $T=6.0$, while static length scales increase modestly above $T_{c}$.

$T=5.25$ and then $T=5.0$. The maximum occurs near $T=6.0$, which is also the temperature at which deviations from mode-coupling fits appear, see Fig. 2 a.

We have carefully checked that this behavior is not a result of our numerical analysis. A direct visual inspection of the time correlations functions $q_{s}(z, t)$ reveals that the spread of the curves in the slow decay has a maximum at $T=6.0$, so that the non-monotonic temperature behavior in Fig. 2 $2 \mathrm{a}$ is not an artifact of our fits, but is a genuine effect (see Supplementary Material, Fig. SM-2). In addition, we found very similar results for the collective relaxation time, $\tau_{c}(z, T)$, which further shows that this non-monotonic behavior does not sensitively depend on the considered observable. Thus, these results give us direct evidence that the relaxation processes responsible for spatial dynamic correlations have a non-monotonic temperature behavior. To our knowledge, all previous numerical and experimental studies of spatially heterogeneous dynamics have reported spatial correlations which grow as the temperature is decreased and dynamics slows down ${ }^{3}-\underline{7}, 18$.

In Fig. 3 we summarize the temperature dependence of the static and dynamic length scales identified above. The static length scales show a modest but steady and monotonic growth with decreasing temperature, which seems to become more pronounced below $T \approx 6.0$. These are natural findings from the RFOT theory perspective $\mathrm{e}^{11,13,16}$, in the sense that static correlations should only become prominent below $T_{c}$. We also include in Fig. 3 the dynamic length scales, which have a striking local maximum near $T=6.0$. A comparison with Fig. 2a strongly suggests to interpret this maximum in the context of RFOT theory in which a dynamic critical point at $T_{c}$ exists in the mean-field limit (but which is avoided in finite dimensions) whereas activated dynam- ics governed by growing static correlations appears at low temperatures. Using RFOT theory Stevenson et al. have suggested that around $T_{c}$ the cooperative domains should indeed change shape, in that they have an open structure above $T_{c}$ and a more compact structure below $T_{c} \underline{26}$. Our findings may be viewed as a striking confirmation of this scenario. Although a gradual change from 'flow-like' to 'hopping' motion was often invoked in the past $\underline{\underline{8}}$, mainly to rationalize successes and failures of the mode-coupling theory, our results provide a very direct, microscopic evidence of a change of relaxation mechanism which involves no theoretical fitting or indirect interpretation.

Although the presented results have been obtained for a system in the presence of pinned disorder, we emphasize that they reflect the behavior of the liquid in the bulk, since the nature of the boundary condition does not affect averaged static properties. No non-monotonic dynamic length scale has been detected so far in bulk systems (leaving out systems that show also anomalies in their thermodynamics), but we think that this is only related to the fact that previous measurements (such as four point correlation function $\left.\chi_{4}{ }^{3,4}\right)$ only provide a coarse representation of spatial correlations, since they mainly probe the total number of 'fast relaxing particles' and not the details of the shape of the relaxing regions ${ }^{4}$. Therefore the present results should not be taken as a contradiction to previous experiments and simulations, but as a new insight into the relaxation mechanism. We hope that in the future new experimental techniques will allow to detect the non-monotonic $T$-dependence of $\xi^{\text {dyn }}$ also in real molecular systems.

If we naively extrapolate our results to lower temperatures, we find a temperature below which static correlations become larger-ranged than dynamic ones, a situation which is physically not very meaningful. Thus we are led to speculate that at much lower temperatures, dynamic length scales should exhibit an upturn, and perhaps become slaved to the static ones, as in the AdamGibbs picture ${ }^{\underline{9}}$ and the scaling regime of RFOT theory 11 . However, studying numerically this final regime is at present too difficult. We suggest that experimental work is needed to resolve these issues further. Our study also suggests that investigations of confined systems should be revisited in both simulations and experiments, and the mode-coupling crossover studied more extensively in glassformers with different fragility. However, since the model investigated here has no unusual features regarding the relaxation dynamics 24 , we expect our results to apply also to other simple models such as hard spheres, Lennard-Jones-like systems, or soft spheres.

Methods- We study an equimolar binary mixture of harmonic spheres ${ }^{24}$ with diameter ratio 1.4, and interactions between particle $i$ and $j$ given by

$V_{i j}\left(\left|\vec{r}_{i}-\vec{r}_{j}\right|\right)=\frac{\varepsilon}{2}\left(1-\left|\vec{r}_{i}-\vec{r}_{j}\right|^{2} / \sigma_{i j}^{2}\right)^{2} \quad$ if $\quad\left|\vec{r}_{i}-\vec{r}_{j}\right|<\sigma_{i j}$,

where $\sigma_{11} \equiv \sigma$ is the unit of length, $\sigma_{12}=1.2$, and 
$\sigma_{22}=1.4$. The total number of particles is 4320 and all of them have the same mass $m$. Time is expressed in units of $\sqrt{m \sigma^{2} / \varepsilon}$ and temperature in units of $10^{-4} \varepsilon$, setting the Boltzmann constant $k_{B}=1.0$. We have used a rectangular box of size $L_{x}=L_{y}=13.68$ and $L_{z}=34.2$, yielding a number density $\rho=0.6749715$. This system size is sufficiently large to avoid finite size effects. The equations of motion have been integrated with the velocity form of the Verlet algorithm. The longest runs extended over 830 million time steps, which took about 6 weeks of CPU time on a high end processor. In order to improve the statistic of the results we have averaged over
10-30 independent walls. The total amount of computer time to obtain the described results was therefore around 7 years. In practice, to prevent particles to penetrate in the frozen half-space, we introduced at the two surfaces of the frozen slice an infinitely hard wall, and have checked that this frozen geometry has negligible influence on the structure of the fluid. (See also Supplementary Material.) Note that we are investigating here a dynamical equilibrium, i.e. all fluid particles can leave their initial positions and explore the whole confined space, thus assuring thermodynamic equilibrium conditions.
1 P.G. Debenedetti and F. H. Stillinger, Supercooled liquids and the glass transition, Nature 410, 259 (2001).

${ }^{2}$ K. Binder and W. Kob, Glassy materials and disordered solids, 2nd edition (World Scientific, Singapore, 2011).

3 M. D. Ediger, Spatially Heterogeneous Dynamics in Supercooled Liquids, Annu. Rev. Phys. Chem. 51, 99 (2000).

4 Dynamical heterogeneities in glasses, colloids, and granular media, Eds.: L. Berthier, G. Biroli, J.-P. Bouchaud, L. Cipelletti, and W. van Saarloos, (Oxford University Press, 2011).

5 C. Bennemann, C. Donati, J. Baschnagel, and S. C. Glotzer, Growing range of correlated motion in a polymer melt on cooling towards the glass transition, Nature 399, 246 (1999).

6 L. Berthier, G. Biroli, J.-P. Bouchaud, L. Cipelletti, D. El Masri, D. L'Hôte, F. Ladieu, and M. Pierno, Direct Experimental Evidence of a Growing Length Scale Accompanying the Glass Transition, Science 310, 1797 (2005).

7 C. Dalle-Ferrier, C. Thibierge, C. Alba-Simionesco, L. Berthier, G. Biroli, J.-P. Bouchaud, F. Ladieu, D. L'Hôte, and G. Tarjus, Spatial correlations in the dynamics of glassforming liquids: Experimental determination of their temperature dependence, Phys. Rev. E 76, 041510 (2007).

8 W. Götze, Complex dynamics of glass-forming liquids: A mode-coupling theory (Oxford University Press, Oxford, 2008).

9 G. Adam and J. H. Gibbs, On the temperature dependence of cooperative relaxation properties in glass-forming liquids, J. Chem. Phys. 43, 139 (1965).

10 D. Chandler and J. P. Garrahan, Dynamics on the Way to Forming Glass: Bubbles in Space-Time, Annu. Rev. Phys. Chem. 61, 191 (2010).

11 T. R. Kirkpatrick, D. Thirumalai, and P. G. Wolynes, Scaling concepts for the dynamics of viscous liquids near an ideal glassy state, Phys. Rev. A 40, 1045 (1989).

12 X. Y. Xia and P. G. Wolynes, Fragilities of liquids predicted from the random first order transition theory of glasses, Proc. Natl. Acad. Sci. USA 97, 2990 (2000).

13 S. Franz and A. Montanari, Analytic determination of $d y$ namical and mosaic length scales in a Kac glass model, J. Phys. A: Math. Theor. 40, F251 (2007).

14 T. R. Kirkpatrick and D. Thirumalai, Dynamics of the structural glass transition and the $p$-spin interaction spin glass model, Phys. Rev. Lett. 58, 2091 (1987).

15 G. Biroli and J. P. Bouchaud, Diverging length scale and upper critical dimension in the Mode-Coupling Theory of the glass transition, Europhys. Lett. 67, 21 (2004).

${ }^{16}$ G. Biroli and J.-P. Bouchaud, The Random First-Order Transition Theory of Glasses: A critical assessment, to appear in a book on RFOT edited by V. Lubchenko and P.W. Wolynes, arXiv:0912.2542

17 D. Coslovich and G. Pastore, Understanding fragility in supercooled Lennard-Jones mixtures. I. Locally preferred structures, J. Chem. Phys. 127, 124504 (2007).

18 H. Tanaka, T. Kawasaki, H. Shintani, and K. Watanabe, Critical-like behavior of glass-forming liquids, Nature Materials 9, 324 (2010).

19 A. Montanari and G. Semerjian, Rigorous inequalities between length and time scales in glassy systems, J. Stat. Phys. 125, 22 (2006).

20 J.-P. Bouchaud and G. Biroli, On the Adam-GibbsKirkpatrick-Thirumalai-Wolynes scenario for the viscosity increase in glasses, J. Chem. Phys. 121, 7347 (2004).

21 G. Biroli, J.-P. Bouchaud, A. Cavagna, T. S. Grigera, and P. Verrocchio, Thermodynamic signature of growing amorphous order in glass-forming liquids, Nature Phys. 4, 771 (2008).

22 P. Scheidler, W. Kob, and K. Binder, Cooperative motion and growing length scales in supercooled confined liquids, Europhys. Lett. 59, 701 (2002).

${ }^{23}$ K. Watanabe, T. Kawasaki, and H. Tanaka, Structural origin of enhanced slow dynamics near a wall in glass-forming systems Nature Materials 10, 511 (2011).

24 L. Berthier and T. A. Witten, Compressing nearly hard sphere fluids increases glass fragility, EPL 86, 10001 (2009).

25 G. Parisi, On the replica scenario for the glass transition, arXiv:0911.2265

26 J. D. Stevenson, J. Schmalian, and P.G. Wolynes The shapes of cooperatively rearranging regions in glass-forming liquids Nature Phys., 2, 268 (2006).

Acknowledgments We thank G. Biroli and A. Cavagna for fruitful exchanges about this work, and the Région Languedoc-Roussillon (L.B.), ANR DYNHET (L.B. and W.K.), and MICINN (Project: MAT200913155-C04-02) and Junta de Andalucía (Project: P07FQM-02496) (S.R.V.) for financial support. W.K. is member of the Institut universitaire de France. 


\section{Supplementary Material}

Influence of the amorphous wall on the local structure of the system

In order to understand better how the presence of the amorphous wall influences the local structure of the confined liquid we show in Fig.-SM-1 a slice of the system orthogonal to the confining walls. The two panels correspond to the two temperature $T=30$ and $T=4.5$.

This figure was obtained by making a run that is about 100 times longer than the $\alpha$-relaxation time in the bulk $\left(=\tau_{s}^{\text {bulk }}(T)\right)$ and by superimposing snapshots of the location of the particles every $\tau_{s}^{\text {bulk }}$. In order to avoid overcrowding, only the particles in a slice of thickness $\Delta x=1.0$ are shown. From this figure one can see that in the center of the box the snapshots start to fill up the space, i.e. the density of the particles becomes uniform, as it should be in a bulk liquid. In contrast to this, the presence of the walls influences the local density. However, as one can demonstrate analytically, see Ref. [27] the structure averaged parallel to the wall is independent of $z$, i.e. is the one of the bulk if one has i) either an infinitely extended wall, or ii) makes an average over sufficiently many independent realizations of the wall.

Comparing panels a) and b) we see that the range over which one finds an influence of the amorphous walls on the local structure of the confined liquid does indeed grow with decreasing temperature, and in the manuscript we demonstrate that this influence decreases exponentially with $z$ with a temperature dependent prefactor (figure 1b). To quantify the distance over which the wall (locally) modifies the density profile, it is reasonable to use $\xi^{\text {stat-int }}$, instead of $\xi^{\text {stat }}$, since the former length takes into account also the prefactor of the mentioned exponential.

Time dependence of the overlap at fixed distance from the wall

In order to show that the non-monotonic temperature dependence of the dynamic length scale $\xi_{s}^{\text {dyn }}$ is not just an effect of the way we have extracted this length scale from the data, and that the decrease is present at several temperatures, we show in Fig. SM-2 the time dependence of the self-overlap $q_{s}(z, t)$ at fixed value of $z$ and several temperatures. One recognizes that at high temperatures, see curves for $T=10$, the curves for the different values of $z$ almost coincide, which implies that the length scale over which the dynamics is influenced by the amorphous walls is very small. With decreasing temperature, curves for $T=6.0$, one sees a significant spread of the curves in the $\alpha$-regime, indicating that $\xi_{s}^{\text {dyn }}$ has increased. It the temperature is lowered even further $(T=5.25$ and $T=5.0)$ this spread is less pronounced than at $T=6.0$, i.e. the $\xi_{s}^{\text {dyn }}$ has decreased again. From this figure we thus can conclude that the non-monotonic $T$-dependence of $\xi_{s}^{\mathrm{dyn}}$ is not an artifact
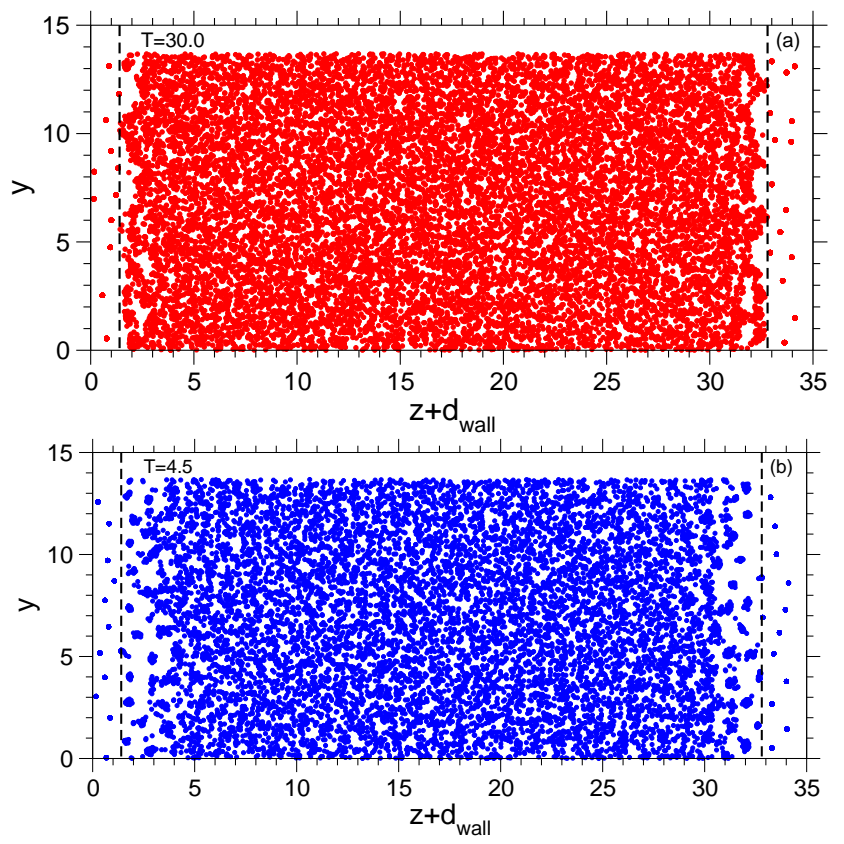

FIG. 4: Figure SM-1: Superposition of snapshots of the system at $T=30.0$ and $T=4.5$ (panel (a) and (b), respectively). The vertical dashed lines show the location of the hard walls. Only particles in the slab $0 \leq x_{i} \leq 1.0$ are shown. Individual snapshots are separated by about $\tau_{s}^{\text {bulk }}(T)$ and the total time is about $100 \tau_{s}^{\text {bulk }}(T)$.

of the way we have analyzed the data and that it is seen directly from the time correlation functions at all temperatures below $T=6.0$.

Determination of the critical temperature of mode-coupling theory

In Fig. 2a of the paper we show an Arrhenius plot of the relaxation times for the system of harmonic spheres. Also included in this graph are fits with the predicted power-laws of mode-coupling theory [28]. Following standard practice in this field, these fits have been obtained in the following way: One assumes a value of $T_{c}$ and makes a $\log -\log$ plot of $\tau_{s}^{\text {bulk }}$ vs. $T-T_{c}$, using of course only data point with $T>T_{c}$. Mode-coupling theory predicts that

$$
\tau_{s}^{\text {bulk }} \propto\left(T-T_{c}\right)^{-\gamma},
$$

where the exponent $\gamma$ is larger than 1.5, and has for hard-sphere-like systems usually a value between 2.0 and 3.0 [8]. By choosing the value of $T_{c}$ in such a way as to rectify the $\tau_{s}^{\text {bulk }}(T)$ data in a large range, one can determine $T_{c}$. In Fig. SM-3 we show the result of such an adjustment for the large particles and we see that the power-law describes the data over about three decades. We find that for our system the exponent $\gamma$ is 2.8, i.e. 


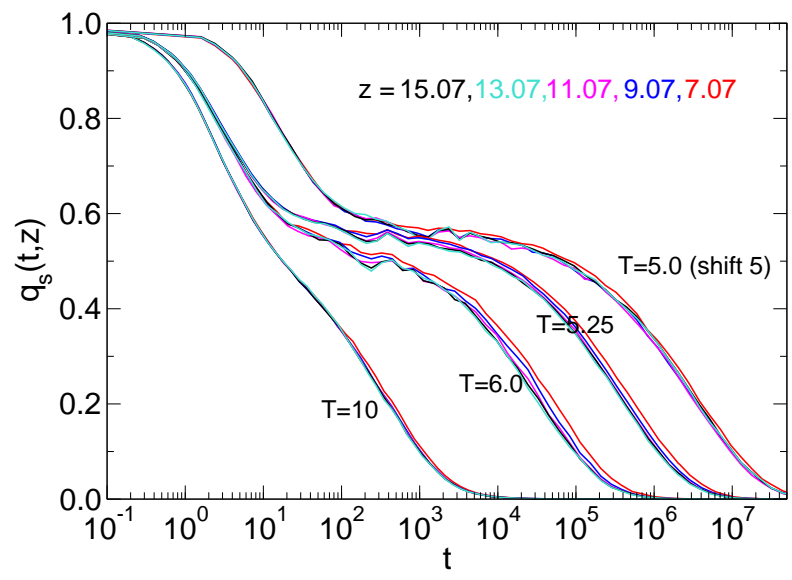

FIG. 5: Figure SM-2: Time dependence of the self-overlap $q_{s}(z, t)$ for selected values of $z$. The temperatures are $T=$ $10.0,6.0,5.25$, and 5.0. The curves for $T=5.0$ have been shifted to the right by a factor of 5.0 in order to avoid overcrowding. Note that in the $\alpha$-relaxation regime the spread of the curves is larger for $T=6.0$ than for the other temperatures, thus showing the non-monotonic temperature dependence of the length scale $\xi_{s}^{\mathrm{dyn}}(T)$.

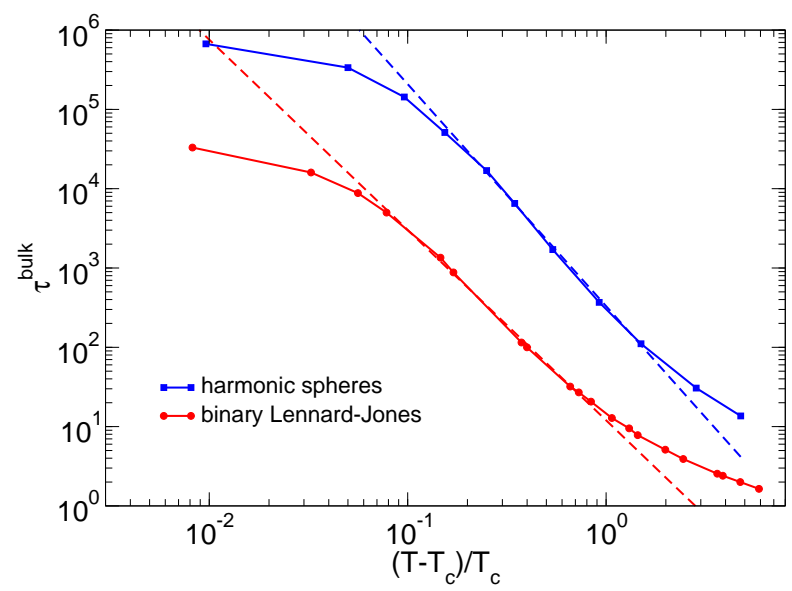

FIG. 6: Figure SM-3: Relaxation time for the bulk as a function of $T-T_{c}$ where $T_{c}$ has been determined by trying to rectify the data. The squares are for the large particles of the harmonic spheres and the circles for the majority particles of the binary Lennard-Jones mixture from Ref. [29]. a value that is very similar to the ones that are found for other glass-forming systems. For the sake of comparison we have included in the graph also the data for the binary Lennard-Jones mixture of Kob and Andersen, a system which has been found to be described very well by mode-coupling theory $[28,29]$. The comparison between the data for the Lennard-Jones system with the one from the harmonic spheres shows that the power-law of modecoupling theory describes the relaxation times well over the same number of decades in $\tau_{s}^{\text {bulk }}$. Therefore we can conclude that also for the system of harmonic spheres the value of $T_{c}$ can be estimated with good accuracy.

We also emphasize that the results we presented here that concern the self-quantity $\left(q_{s}(z, t)\right.$ and $\left.\tau_{s}^{\text {bulk }}\right)$ are confirmed by the ones for the collective quantities $\left(q_{c}(z, t)\right.$ and $\left.\tau_{c}^{\text {bulk }}\right)$, i.e. there is no relevant difference between these two observables as far as relaxation times are concerned.

\section{References}

[27] P. Scheidler, W. Kob, and K. Binder, The relaxation dynamics of a supercooled liquid confined by rough walls, J. Phys. Chem. 108, 6673 (2004).

[28] W. Kob and H. C. Andersen, Testing modecoupling theory for a supercooled binary Lennard-Jones mixture: The van Hove correlation function Phys. Rev. E 51, 4626 (1995).

[29] M. Nauroth and W. Kob, A quantitative test of the mode-coupling theory of the ideal glass transition for a binary Lennard-Jones system Phys. Rev. E 55, 657 (1997). 